\title{
Phase Structure of Kerr-AdS Black Hole
}

\author{
Tsai, Yu-Dai* \\ Department of Physics, National Tsing Hua University, Hsinchu, Taiwan 300, R.O.C. \\ X. N. Wu \\ Institute of Mathematics, Academy of Mathematics and Systems Science, \\ Chinese Academy of Sciences, Beijing 100190, P.R.C. \\ Yi Yang $\left.\right|^{\ddagger}$ \\ Department of Electrophysics, National Chiao \\ Tung University, Hsinchu, Taiwan 300, R.O.C.
}

(Dated: December 12, 2011)

\begin{abstract}
We study the critical phenomena of Kerr-AdS black hole. Phase structures are observed at different temperatures, $T_{L}, T_{c 1}$ and $T_{c 2}$ with various features. We discuss the thermal stability considering the isothermal compressibility and how phase transitions related to each other. The asymptotic value of the angular momentum also has an implication on separating stable and unstable part. Near critical temperature $T_{c 1}$, the order parameter is determined to calculate the critical exponents. All the critical exponents $(\alpha, \beta, \gamma, \delta)=\left(0, \frac{1}{2}, 1,3\right)$ are identical to that of mean field systems. We plot the phase diagram near this critical point, and discuss the scaling symmetry of the free energy.
\end{abstract}

\footnotetext{
*Electronic address: b94201020@ntu.edu.tw

${ }^{\dagger}$ Electronic address: wuxn@amss.ac.cn

${ }^{\ddagger}$ Electronic address: yiyang@mail.nctu.edu.tw
} 


\section{INTRODUCTION}

During the last few decades, black hole thermodynamics has been playing the role of a "thinking experiment" to understand quantum gravity. The discovery of Hawking radiation shows that the analogy between black hole mechanical laws and the laws of thermodynamics is physically meaningful. Based on this analogy, Davis pioneered to consider the phase transition of RN black holes [13. Hawking and Page later investigated the phase transition of Schwarzschild-AdS black holes in [18]. Following their path-breaking research, many works have been done along this direction and rich phase structures have been discovered [1, 4-6, 8, 10, 19]. The later established AdS/CFT duality [20, 29] further inspired people to focus on the asymptotically anti-de Sitter (AdS) black holes. Critical phenomena were discovered in asymptotically AdS black holes (see [2, 3, 7, 9, 12, 21, 25, 30]).

In [12], Chamblin et al. studied the phase structures of RN-AdS black hole. They identified a critical point in RN-AdS black hole by considering the divergence of heat capacity. Near this critical point, the behavior of isotherms are similar to that of van der Waals liquid/gas system. However, the critical exponents of RN-AdS black hole are different from that of the van der Waals case as it has been shown in [30]. A detailed investigation of the phase structure of Kerr-AdS black hole is needed to be compared to the previous results.

We hope the report of critical phenomena can help us to achieve the ultimate goal of finding a microscopic description of the black hole phase structure.

Another motivation of this work came from the ideas of holographic superconductors (see [16, 17]) and their rotating extension (see [26]). In [26], Sonner studied the superconducting phase transition on the boundary of Kerr-Newman-AdS black hole. The phase structure of the background field may affect some properties of the rotating holographic superconductor. And the knowledge of phase transitions of Kerr-AdS and RN-AdS black holes could be essential to fully understand the holographic superconductors.

In this paper, we study the phase structure of Kerr-AdS black hole. Rich phase structure were discovered at three diverse critical temperatures, and this multi-critical phenomenon in Kerr-AdS black hole has not been carefully discussed in the previous literatures.

To begin with, we briefly discuss the phase structure by plotting the isotherm and discribe the three critical temperatures. We then detailedly discuss the critical behavior of each isotherm near the three critical temperature $T_{L}, T_{c 1}$ and $T_{c 2}$, respectively. We determind 
the asymptotic value of the angular momentum, which is important to understand the thermal stability of the Kerr-AdS black hole.

At a certain temperature $T_{c 1}$, we discovered van der Waals-like phase transition. Unlike the previous case in [25, 30], the critical exponents of Kerr-AdS black hole are found identical to the van der Waals liquid/gas system and the Weiss ferromagnet. It provides a strong evidence that Kerr-AdS black hole system belongs to the universality class which contains these two systems. We also discuss the scaling symmetry of the free energy near this critical point.

The paper is organized in the following manner. In section [I] we introduce the necessary basics of Kerr-AdS black hole and define its thermodynamics quantities. The asymptotic behavior of angular velocity is briefly discussed. The critical isotherms are plotted in section III, in which we briefly discuss the phase structure of Kerr-AdS black hole at $T_{L}, T_{c 1}$ and $T_{c 2}$,

respectively. The asymptotic angular velocity has an implication on the thermal stability of Kerr-AdS black hole through the phase transition happens at $T_{c 2}$ In section IV, we calculate the critical exponents of the newly discovered critical point at $T_{c 1}$ can compare it to the well-known phase transition systems, such as Weiss ferromagnet system and van der Waals liquid/gas system. We make analogy of the free energy and discuss its scaling symmetry. The paper is concluded in section $\mathrm{V}$ with discussions and future outlooks.

\section{THERMODYNAMICS OF KERR-ADS BLACK HOLE}

Kerr-AdS black hole is a rotating black hole in AdS space-time. The exact Kerr-AdS black hole solution of the Einstein equations is given by [11] in the Boyer-Lindquist coordinates as

$$
d s^{2}=-\frac{\Delta_{r}}{\Sigma}\left(d t-\frac{a \sin ^{2} \theta}{\Xi} d \phi\right)^{2}+\frac{\Sigma}{\Delta_{r}} d r^{2}+\frac{\Sigma}{\Delta_{\theta}} d \theta^{2}+\frac{\Delta_{\theta} \sin ^{2} \theta}{\Sigma}\left(a d t-\frac{r^{2}+a^{2}}{\Xi} d \phi\right)^{2},
$$

where

$$
\begin{aligned}
& \Delta_{r}=\left(r^{2}+a^{2}\right)\left(1+\frac{r^{2}}{l^{2}}\right)-2 M r, \quad \Xi=1-\frac{a^{2}}{l^{2}}, \\
& \Delta_{\theta}=1-\frac{a^{2}}{l^{2}} \cos ^{2} \theta, \quad \Sigma=r^{2}+a^{2} \cos ^{2} \theta .
\end{aligned}
$$


Here $M$ is the mass of Kerr-AdS black hole and $a$ is the rotational parameter related to the angular momentum of the black hole. If we take $a=0$, the above metric reduces to the Schwarzschild metric.

The curvature radius $l$ is related to the negative cosmological constant $\Lambda$ by $\Lambda=-3 l^{-2}$. And the radius of horizon $r_{+}$is defined by taking $\Delta_{r}=0$, i.e.

$$
\left(r_{+}^{2}+a^{2}\right)\left(1+\frac{r_{+}^{2}}{l^{2}}\right)-2 M r_{+}=0
$$

So the area of event horizon $A$ is

$$
A=\frac{4 \pi\left(r_{+}^{2}+a^{2}\right)}{\Xi} .
$$

\section{A. Thermodynamic Quantities of Kerr-AdS Black Hole}

The thermodynamic quantities of the Kerr-AdS black hole [14] can be expressed in terms of the radius of horizon $r_{+}$, the rotational parameter $a$ and the cosmological constant $\Lambda$ $\left(\Lambda=-3 / l^{2}\right)$. The Hawking temperature of Kerr-AdS black hole is now

$$
T=\frac{3 r_{+}^{4}+\left(a^{2}+l^{2}\right) r_{+}^{2}-l^{2} a^{2}}{4 \pi l^{2} r_{+}\left(r_{+}^{2}+a^{2}\right)},
$$

While the Bekenstein-Hawking entropy is $S=A / 4$.

Consider the first law of Kerr-AdS black hole [14]

$$
d E=T d S+\Omega d J
$$

One have to choose the angular velocity $\Omega$ measured relative to a frame which is non-rotating at infinity. This quantity is defined as

$$
\Omega=\frac{a\left(1+r_{+}^{2} l^{-2}\right)}{r_{+}^{2}+a^{2}} .
$$

Now for Eq.(6), one has the "physical" mass (or energy) $E$ and angular momentum $J$ defined as

$$
E=\frac{M}{\Xi^{2}}, \quad J=\frac{M a}{\Xi^{2}} .
$$


By solving Eq.(3) to determine $M$, we have

$$
J=\frac{a\left(1+\frac{r_{+}^{2}}{l^{2}}\right)\left(a^{2}+r_{+}^{2}\right)}{2 r_{+}\left(1-\frac{a^{2}}{l^{2}}\right)^{2}} .
$$

\section{B. Equation of State and Asymptotic Behavior of $\Omega$ in Large $J$}

Because $\Lambda$ is a constant, we can rescale each quantity to simplify the expressions and calculations,

$$
\begin{aligned}
& l T \rightarrow T, \quad l \Omega \rightarrow \Omega, \\
& \frac{J}{l} \rightarrow J \quad, \frac{M}{l} \rightarrow M, \frac{a}{l} \rightarrow a, \frac{r_{+}}{l} \rightarrow r_{+} .
\end{aligned}
$$

Thus $J, \Omega$, and $T$ become

$$
\begin{aligned}
J & =\frac{a\left(1+r_{+}^{2}\right)\left(a^{2}+r_{+}^{2}\right)}{2 r_{+}\left(1-a^{2}\right)^{2}}, \\
\Omega & =\frac{a\left(1+r_{+}^{2}\right)}{r_{+}^{2}+a^{2}} \\
T & =\frac{3 r_{+}^{4}+a^{2} r_{+}^{2}+r_{+}^{2}-a^{2}}{4 \pi r_{+}\left(r_{+}^{2}+a^{2}\right)} .
\end{aligned}
$$

Here we have three equations which govern the phase structure of the Kerr-Ads black hole, which can be regarded as "equations of state". The functions $\Omega, J$ and $T$ are complicatedly

dependent on $r_{+}$and $a$, so that it is difficult to solve $r_{+}(J, \Omega)$ and $a(J, \Omega)$ analytically to get the equation of state $T=T(\Omega, J)$ directly.

To begin with, we solve $a$ as a function of $T$ and $r_{+}$to get

$$
a=\sqrt{\frac{r_{+}^{2}+3 r_{+}^{4}-4 \pi r_{+}^{3} T}{1-r_{+}^{2}+4 \pi r_{+} T}} .
$$

By putting $a$ back into (11) and (12), we obtain the following parametrized expressions for $J$ and $\Omega$,

$$
\begin{aligned}
J & =\frac{r_{+}^{2} \sqrt{\left(1-r_{+}^{2}+4 \pi r_{+} T\right)\left(1+3 r_{+}^{2}-4 \pi r_{+} T\right)}}{\left(1-3 r_{+}^{2}+4 \pi r_{+} T\right)^{2}} \\
\Omega & =\frac{\sqrt{\left(1-r_{+}^{2}+4 \pi r_{+} T\right)\left(1+3 r_{+}^{2}-4 \pi r_{+} T\right)}}{2 r_{+}}
\end{aligned}
$$


These two parametrized equations allow us to discuss the asymptotic behavior of $\Omega$ on an isotherm when $J$ goes to infinity. For a fixed $T$, angular momentum $J$ goes to infinity as $r_{+} \rightarrow \frac{1}{3}\left(2 \pi T+\sqrt{3+4 \pi^{2} T^{2}}\right)$. Taking $r_{+}$to this value, we get

$$
\Omega \rightarrow 1
$$

Thus we have the asymptotic value of $\Omega$ as one when $J$ goes to infinity.

We name this asymptotic value $\Omega=1$ as $\Omega_{c 2}$ because it relates to the critical temperature $T_{c 2}$. This critical behavior at $T_{c 2}$ will be further discussed in section IIIB, In the next section, we will plot the isotherms to study the phase structure of Kerr-AdS black hole.

\section{CARVING THE PHASE STRUCTURE}

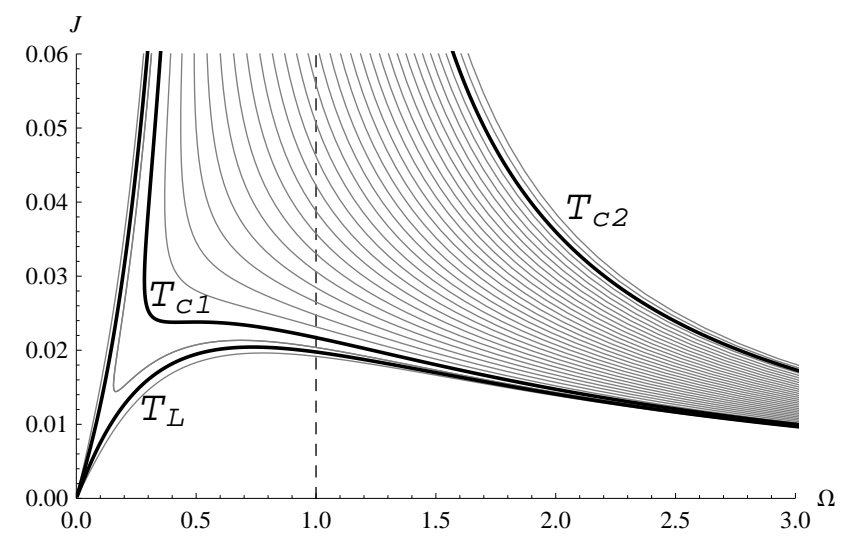

FIG. 1: The isotherms on the $(J, \Omega)$ space. Three isotherms has been labeled by their temperatures, $T_{L}, T_{c 1}$ and $T_{c 2} . T_{L}>T_{c 1}>T_{c 2}$.

We present the isotherms at various temperatures in FIG. 1. Fos each fixed temperature $T$, we vary $r_{+}$to get an isotherm of $(J, \Omega)$ by using Eq.(15) and Eq. (16).

The first apparent feature we observed of these isotherms is that above a certain temperature $T_{L}=0.2757$, the isotherms become $L$-shaped with their cusp located on $(J, \Omega)=(0,0)$, as one can see in Fig. 1. The Kerr-AdS black hole reduces to Schwarzchild-AdS black hole at this point. The isotherm has positive slope near the $(0,0)$ point, which makes this part thermally unstable as far as the isothermal compressibility $\kappa_{T}$ being considered. This $L$ shaping critical phenomena also destroy the phase structure of $T_{c 1}$ at $T_{c 1 \prime}\left(T_{c 1}<T_{c 1}<T_{L}\right)$, as we will discuss in section IV. 
There are two other isotherms being specifically labeled in FIG. 1 with their temperatures $T_{c 1}$ and $T_{c 2}$. Two different critical phenomena occur at lower temperatures. To see the phase structure at $T_{c 1}$ and $T_{c 2}$ clearly, we plot the isotherms around the critical temperatures in different scales in FIG,2 and 3 ,

\section{A. Phase Structure Near Critical Point at $T_{c 1}$}

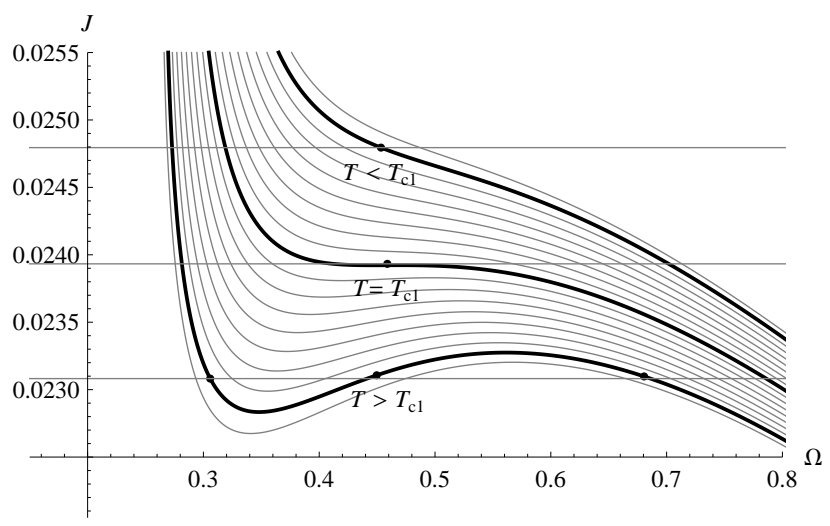

FIG. 2: The critical isotherms near the critical temperature $T_{c 1}$.

First we discuss the phase transition taking place at $T_{c 1}=0.270$. This temperature is determined by solving the standard critical point equations (see [23]),

$$
\left(\frac{\partial J}{\partial \Omega}\right)_{T_{c 1}}=0, \quad\left(\frac{\partial^{2} J}{\partial \Omega^{2}}\right)_{T_{c 1}}=0 .
$$

By using the above equations of state Eq. (15) and Eq.(16), we determine $\left(\Omega_{c 1}, J_{c 1}\right)=$ $(0.459,0.024)$. The isotherms around this critical point are plotted in FIG. 2. At this critical point, the isothermal compressibility $\kappa_{T}=\left(\frac{\partial \Omega}{\partial J}\right)_{T}$ and the specific heat $C_{J}$ both diverge. And above $T_{c 1}$, a single $J$ corresponds to multiple $\Omega$ s for each fixed temperture.

FIG 2 is similar to the liquid/gas PVT diagram [23]. This van der Waals-like phase transition can be clearly visualized as we choose the $J \rightarrow P, \Omega \rightarrow V$ correspondence. We will make a detailed study of this critical point in section IV. 


\section{B. Phase Structure Near Critical Point at $T_{c 2}$}

The other critical temperature, $T_{c 2}=0.159$, is determined when an isotherm has its slope goes to infinity $\left(\kappa_{T} \rightarrow 0\right)$ when $J \rightarrow \infty$. Below the temperature $T_{c 2}$, the isothermal compressibility $\kappa_{T}$ is always negative along each isotherm, which means that the black hole is stable under this consideration. But as one raise the temperature to above $T_{c 2}$, positive isothermal compressibility emerge on the upper part of the curves, making the black hole thermally unstable. We highlights the unstable region as the shadowed region in FIG.3 as well as the critical isotherm at $T_{c 2}$. The $J$ value is plotted on the $\log$ coordinate, while $\Omega$ is on the original coordinate, to better visualize the critical behavior of isotherms near $T_{L}, T_{c 1}$ and $T_{c 2}$.

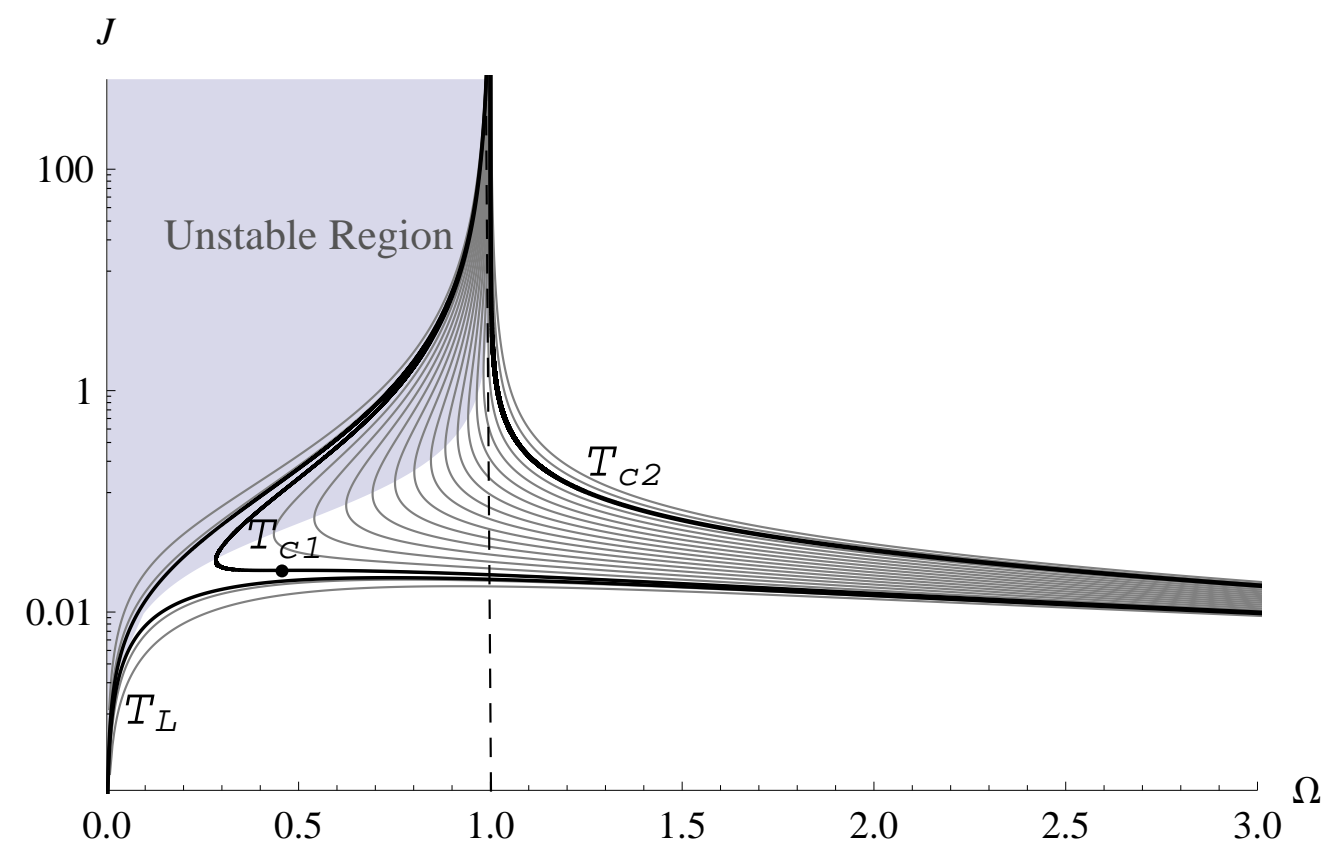

FIG. 3: The diagram contains all three critical phenomena with their features. Here $J$ is plot on the log coordinate.

One can understand the critical behavior at $T_{c 2}$ in another manner. The asymptotic angular velocity, $\Omega_{c 2}$, also marks the critical angular momentum where the points on the isotherms could become thermally unstable in the upper part, as we can see in FIG. 3. On the right side of $\Omega=\Omega_{c 2}$ line, all the isotherms are stable on the upper part. But on the left side of $\Omega=\Omega_{c 2}$, the unstable parts start to emerge on the upper part of isotherms. It is an important feature: Kerr-AdS black hole not only has an asymptotic angular velocity, but 
this asymptotic angular velocity also determine the thermal stability as far as isothermal compressibility is considered.

In the end of this section, we summarize the phase structure of Kerr-AdS black hole with the aid of FIG.3. The isotherm at $T_{L}$ has its cusp located on $(0,0)$. The critical isotherm at $T_{c 1}$ has zero slope at its critical point (the black point in the figure) and then slowly bending downward as $\Omega$ increases. The isotherm at $T_{c 2}$ has infinite slope when $J$ is very large. One can also see the asymptotic/critical value $\Omega_{c 2}=1$ which separates the stable and the unstable regions in FIG 3 .

\section{THE PHASE STRUCTURE NEAR $\mathbf{T}_{c 1}$}

In this chapter, we consider the phase transition which happens above critical temperature $T_{c 1}$. In FIG. 2, this van der Waals-like phase structure can be clearly visualized. This critical point happens when we choose grand canonical (fixed horizon angular velocity $\Omega$ ) ensemble. The critical phenomena has not been discussed carefully in the previous literatures.As pointed out by [24], the phase structure of black holes strongly depends on the choice of the ensembles. We study the quantitative properties of this newly found secondorder phase transition, and compare it to well known systems.

\section{A. Determination of the Critical Point}

The first critical point can be determined by of the following conditions,

$$
\left(\frac{\partial J}{\partial \Omega}\right)_{T}=0, \quad\left(\frac{\partial^{2} J}{\partial \Omega^{2}}\right)_{T}=0
$$

which can be expressed as functions of $r_{+}$and $T$ by Eq.(15) and Eq.(16),

$$
\begin{gathered}
\left(\frac{\partial J}{\partial \Omega}\right)_{T}=\frac{\left(\frac{\partial J}{\partial r_{+}}\right)_{T}}{\left(\frac{\partial \Omega}{\partial r_{+}}\right)_{T}}=J_{\Omega}\left(r_{+}, T\right) \\
\left(\frac{\partial^{2} J}{\partial^{2} \Omega}\right)_{T}=\frac{\left(\frac{\left(\frac{\partial J}{\partial \Omega}\right)_{T}}{\partial r_{+}}\right)_{T}}{\left(\frac{\partial \Omega}{\partial r_{+}}\right)_{T}}=J_{\Omega^{2}}\left(r_{+}, T\right)
\end{gathered}
$$


Solving the above two equations, we find that at the critical point $T=T_{c 1} \equiv 0.270$ and $r_{+}=r_{c 1} \equiv 0.459$. Then we obtain $\left(\Omega_{c 1}, J_{c 1}\right)=(0.459,0.024)$.

\section{B. Order Parameter and Law of Equal Area}

We will define the order parameter to describe the critical behavior near the critical point. Above the critical temperature $T_{c 1}$, there are three points on an isotherm having the same $J$ but with the different $\Omega$ s. Like van der Waals system, one can define an order parameter by Maxwell's equal-area law. As in FIG. 4, we choose a isotherm with $T>T_{c 1}$ and draw a horizontal line which intersect the isotherm at three points $a, m$ and $b$. When the area $\mathrm{A}$ is equal to the area $\mathrm{B}$, the value $\eta=\left(\Omega_{b}-\Omega_{a}\right) / 2$ is defined as the order parameter. This method also allow us to plot the coexistence curve as we present in FIG. 5 . At $T_{c 1 \prime}=0.2735$ (determined numerically), the area A of the left part becomes too small to achieve Maxwell's equal-area law, therefore the phase transition ends at the temperature $T_{c 1 \text {. }}$.

For the mean field systems, spontaneous magnetism is the order parameter in Weiss ferromagnetic system, while the half width of the coexistence curve is the order parameter in the van der Walls system [23].

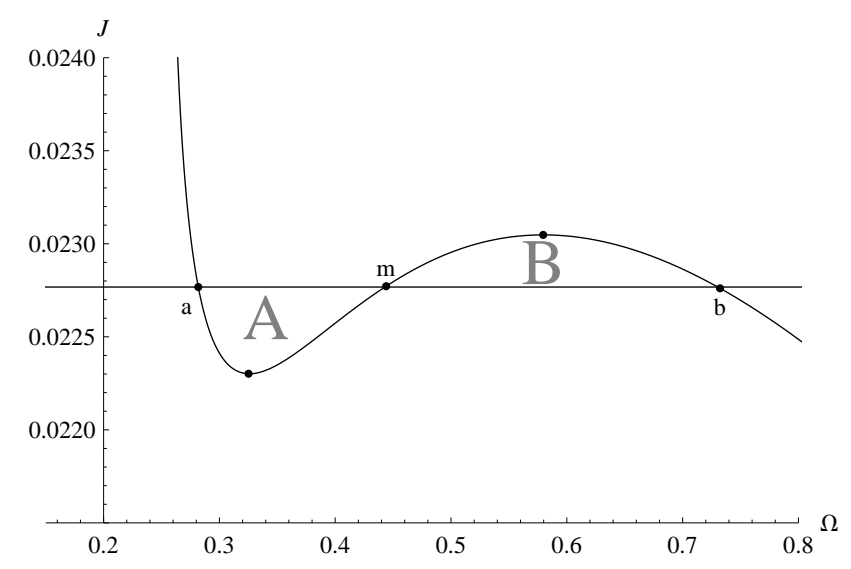

FIG. 4: Applying the equal-area law on an isotherm at a temperature $T>T_{c 1}$.

\section{Critical Exponents}

With the order parameter $\eta=\left(\Omega_{a}-\Omega_{b}\right) / 2$ which we defined in the previous section, we will calculate the following well known critical exponents in this section. 
Degree of critical isotherm:

$$
J-J_{c 1}=A_{\delta}\left|\Omega-\Omega_{c 1}\right|^{\delta} \operatorname{sign}\left(\Omega-\Omega_{c 1}\right), \quad T=T_{c 1} .
$$

Degree of coexistence curve:

$$
\eta=-A_{\beta}\left(T-T_{c 1}\right)^{\beta}, \quad T>T_{c 1} .
$$

Degree of heat capacity $\left(\Omega=\Omega_{c 1}\right)$ :

$$
C_{\Omega}= \begin{cases}A_{\alpha^{\prime}}\left\{-\left(T-T_{c 1}\right)\right\}^{-\alpha^{\prime}}, & T<T_{c 1} \\ A_{\alpha}\left\{+\left(T-T_{c 1}\right)\right\}^{-\alpha}, & T>T_{c 1}\end{cases}
$$

Degree of isothermal compressibility:

$$
\kappa_{T}=\left\{\begin{array}{ll}
A_{\gamma^{\prime}}\left\{-\left(T-T_{c 2}\right)\right\}^{-\gamma^{\prime}}, & T<T_{c 1} \\
A_{\gamma}\left\{+\left(T-T_{c 2}\right)\right\}^{-\gamma}, & T>T_{c 1}
\end{array} .\right.
$$

Following the discussion in [23], the definitions of the degree of isothermal compressibility $\kappa_{T}$ for $T<T_{c 1}$ and $T<T_{c 1}$ are different. For $T<T_{c 1}, \gamma^{\prime}$ is defined along the iso-angular momentum line, i.e., $\Omega=\Omega_{c 1}$; when $T>T_{c 1}, \gamma$ is defined along the coexistence curve, which is illustrated in FIG. 5 .

Next, we will calculate these critical exponents of the phase transitions in Kerr-AdS black hole system one by one.

\section{Degree of Critical Isotherm}

At this critical point $T=T_{c 1}$, the first and second derivatives of $J$ over $\Omega$ satisfy

$$
\left.\left(\frac{\partial J}{\partial \Omega}\right)_{T}\right|_{c 1}=\left.\left(\frac{\partial^{2} J}{\partial \Omega^{2}}\right)_{T}\right|_{c 1}=0
$$

The third order derivative can be calculated as

$$
\left.\left(\frac{\partial^{3} J}{\partial^{3} \Omega}\right)_{T}\right|_{c 1}=-0.539 \neq 0
$$


which is not vanishing, therefore we get $\delta=3$.

\section{Degree of Coexistence Curve}

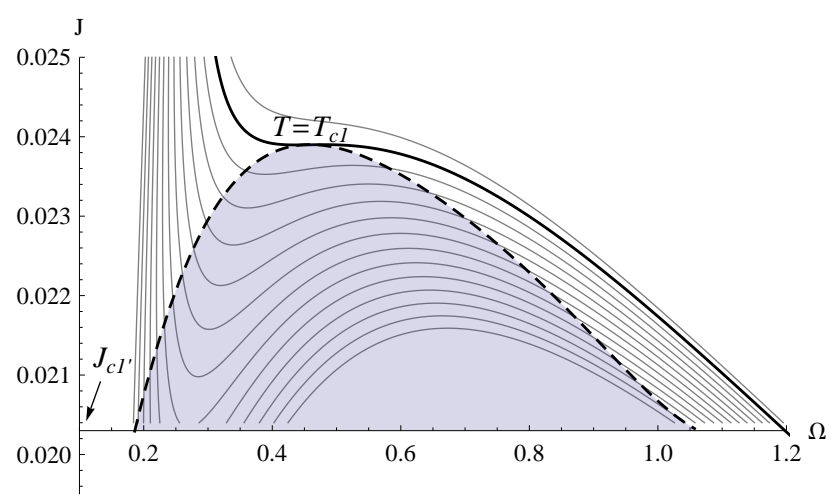

FIG. 5: The dashed curve is the coexistence curve of phase transition above $T_{c 1}$. The coexistence curve ends at $J_{c 1}$, because the stable area is too small to apply the equal area law at the corresponding temperature $T_{c 1}$,

In FIG. 5 we plot the curve of the coexisting states by Maxwell's equal-area law. This curve indicates the temperature dependence of the order parameter right after the order parameter emerges.

We expand $J$ in terms of $\Omega$ and $T$ to the third order as

$$
\begin{array}{cc}
J-J_{c 1} & \left.\approx\left(\partial_{T} J\right)_{\Omega}\right|_{c}\left(T-T_{c 1}\right) \\
& +\left.\left(\partial_{T}\left(\partial_{\Omega} J\right)_{T}\right)_{\Omega}\right|_{c 1}\left(\Omega-\Omega_{c 1}\right)\left(T-T_{c 1}\right) \\
+ & +\left.\frac{1}{2}\left(\partial_{T}\left(\partial_{\Omega}^{2} J\right)_{T}\right)_{\Omega}\right|_{c 1}\left(\Omega-\Omega_{c}\right)^{2}\left(T-T_{c 1}\right) \\
+ & \left.\frac{1}{2}\left(\partial_{T}^{2}\left(\partial_{\Omega} J\right)_{T}\right)_{\Omega}\right|_{c 1}\left(\Omega-\Omega_{c 1}\right)\left(T-T_{c 1}\right)^{2} \\
+\left.\frac{1}{3 !}\left(\partial_{\Omega}^{3} J\right)_{T}\right|_{c 1}\left(\Omega-\Omega_{c 1}\right)^{3}
\end{array}
$$

To simplify the calculation, we define

$$
j=J-J_{c 1}, \quad t=T-T_{c 1}, \quad \omega=\Omega-\Omega_{c 1},
$$

so that

$$
j=c_{10} t+c_{11} \omega t+c_{21} \omega t^{2}+c_{12} \omega^{2} t+c_{03} \omega^{3} .
$$


All coefficients $c_{i j}$ in Eq. (30) can be calculated by the standard partial differentiation. The results are

$$
c_{10}=-1.027, c_{11}=3.684, c_{12}=-15.792, c_{21}=-458.833, c_{03}=-0.090 .
$$

As we will see in the following discussions, the critical exponents are not sensitive to the exact values of these constants.

By applying the conditions of equal angular momentum and equal area,

$$
j\left(\omega_{a}, t\right)=j\left(\omega_{b}, t\right), \quad \int_{\omega_{b}}^{\omega_{a}} \omega d j=0
$$

we found that

$$
\begin{gathered}
-\left(\omega_{a}-\omega_{b}\right)\left[c_{11} t+c_{21} t^{2}+c_{12} t\left(\omega_{a}+\omega_{b}\right)+c_{03}\left(\omega_{a}^{2}+\omega_{a} \omega_{b}+\omega_{b}^{2}\right)\right]=0, \\
-\left(\omega_{a}-\omega_{b}\right)\left[6 c_{11} t\left(\omega_{a}+\omega_{b}\right)+6 c_{21} t^{2}\left(\omega_{a}+\omega_{b}\right)\right. \\
\left.\quad+8 c_{12} t\left(\omega_{a}^{2}+\omega_{a} \omega_{b}+\omega_{b}^{2}\right)+9 c_{03}\left(\omega_{a}^{3}+\omega_{a}^{2} \omega_{b}+\omega_{a} \omega_{b}^{2}+\omega_{b}^{3}\right)\right]=0 .
\end{gathered}
$$

Changing the variables as

$$
\omega_{-} \equiv \omega_{b}-\omega_{a}=\Omega_{b}-\Omega_{a}, \omega_{+} \equiv \omega_{b}+\omega_{a}
$$

one can solve $\omega_{+}$and $\omega_{-}$as

$$
\begin{gathered}
\omega_{+}=-\frac{2 c_{12} t}{3 c_{03}}, \\
\omega_{-}=\sqrt{\frac{-4 c_{11} t+\left(\frac{4 c_{12}^{2}}{3 c_{03}}-4 c_{21}\right) t^{2}}{c_{03}}} .
\end{gathered}
$$

Now we can expand $t$ in term of $\omega_{-}$as

$$
t=-\frac{c_{11} c_{03}}{4 c_{11}^{2}} \omega_{-}^{2}+O\left(\omega_{-}^{4}\right)
$$

to get the order parameter

$$
\frac{\Omega_{b}-\Omega_{a}}{2} \approx A_{\beta}\left(T-T_{c 1}\right)^{\frac{1}{2}}
$$


Thus we $\operatorname{read} \beta=\frac{1}{2}$.

\section{Critical Exponent of Heat Capacity}

We now consider the critical exponent of the heat capacity along the constant angular velocity line $\Omega=\Omega_{c}$. By the black hole thermal dynamical laws, the role of the internal energy $Q$ is played by the black hole mass $E$ of the Kerr-AdS black hole. $E$ is given by Eq. (8) and is rescaled by $\mathrm{Eq}(10)$ as

$$
E=\frac{r_{+}^{3}-r_{+}^{5}+4 \pi r_{+}^{4} T}{\left(1-3 r_{+}^{2}+4 \pi r_{+} T\right)^{2}}
$$

The heat capacity $C_{\Omega}$ can be calculated as

$$
C_{\Omega}=\left.\left(\frac{\partial E}{\partial T}\right)_{\Omega}\right|_{c_{1}}=-2.880 \neq 0
$$

Therefore, $\alpha$ and $\alpha^{\prime}$ are both zero because the heat capacity neither diverges nor vanishes, i.e. $\alpha=\alpha^{\prime}=0$.

\section{Degree of Isothermal Compressibility}

The isothermal compressibility $\kappa_{T}$ is defined as

$$
\kappa_{T}=\left(\frac{\partial \Omega}{\partial J}\right)_{T}
$$

which is divergent at the critical point. To do the Taylor expansion, we consider the inverse of the isothermal compressibility

$$
\kappa_{T}^{-1}=\left\{\begin{array}{ll}
A^{\prime}\left\{-\left(T-T_{c 1}\right)\right\}^{\gamma^{\prime}}, & \left(T<T_{c 1}\right) \\
A\left\{+\left(T-T_{c 1}\right)\right\}^{\gamma}, & \left(T>T_{c 1}\right)
\end{array} .\right.
$$

Using Eq. (30), we have

$$
\kappa_{T}^{-1} \propto\left(\frac{\partial j}{\partial \omega}\right)_{t} \approx c_{11} t
$$


TABLE I: The comparison of critical exponents

\begin{tabular}{ccccc}
\hline Exponent & Weiss/van der Waals & Kerr-AdS $T_{c 1}$ & $\operatorname{Ising}(\mathrm{d}=2)$ & $\operatorname{Ising}(\mathrm{d}=3)$ \\
\hline$\alpha$ & 0 & 0 & 0 & $0.110(5)$ \\
$\beta$ & $1 / 2$ & $1 / 2$ & $1 / 8$ & $0.325+0.0015$ \\
$\gamma$ & 1 & 1 & $7 / 4$ & $1.2405+0.0015$ \\
$\delta$ & 3 & 3 & 15 & $4.82(4)$ \\
\hline
\end{tabular}

The first order dependence is correct for both $T>T_{c 1}$ (along the iso-angular velocity line, on which $\omega=\omega_{c}$ ) and $T<T_{c 1}$ (along coexistence curve, on which $\omega \propto t^{\frac{1}{2}}$ ). Therefore we get $\gamma=\gamma^{\prime}=1$.

\section{Free Energy and Comparison with Mean Field Models}

In Table $[$, we compared the critical exponents for various systems. It is interesting to take the point of view of the mean field theory to look at the results we got in the previous sections.

In Eq. (30), we expanded the equation of state of Kerr-AdS black hole in the vicinity of the critical point. With the definition of $\epsilon=\left(T-T_{c 1}\right) / T_{c 1}$ and the order parameter $\eta=\left(\Omega-\Omega_{c 1}\right) / \Omega_{c 1}$, the equation of state becomes

$$
j=\tilde{c}_{10} \epsilon+\tilde{c}_{11} \epsilon \eta+\tilde{c}_{21} \epsilon^{2} \eta+\tilde{c}_{12} \epsilon \eta^{2}+\tilde{c}_{03} \eta^{3}
$$

where $\tilde{c}_{10}, \tilde{c}_{11}, \tilde{c}_{21}, \tilde{c}_{12}, \tilde{c}_{03}$ are constant coeeficients.

The equation of state can be derived from the free energy,

$$
g(j, \epsilon, \eta)=g_{0}(j, \epsilon)-\left(j-c_{10} \epsilon\right) \eta+\left(c_{11} \epsilon+c_{21} \epsilon^{2}\right) \frac{1}{2} \eta^{2}+c_{12} \epsilon \frac{1}{3} \eta^{3}+c_{03} \frac{1}{4} \eta^{4}
$$

Now we compare our result with some mean field systems.

The first system is Weiss ferromagnet system, see [15]. The equation of state is

$$
\frac{H}{k_{B} T}=M(1-\tau)+M^{3}\left(\tau-\tau^{2}+\tau^{3} / 3+\cdots\right)
$$

where $M$ is the average magnetic moment, $H$ is the external field, and $\tau^{-1}$ is defined as 
$T / T_{c}$.

In terms of $\epsilon=\left(T-T_{c}\right) / T_{c}$ as well as order parameter $\eta=M$, this equation of state can be written as

$$
\frac{H}{k_{B} T}=\eta \epsilon+\eta^{3}+O\left(\epsilon \eta^{3}\right),
$$

which can be derived from the free energy $\Gamma$ as,

$$
\Gamma(\eta, T, H)=\Gamma_{0}(T, H)-\frac{\eta H}{k_{B} T}+\frac{\epsilon \eta^{2}}{2}+\frac{1}{4} \eta^{4} .
$$

Another mean field system we will consider is van der Waals gas/liquid system, see also [15]. The equation of state is

$$
\pi=\frac{P-P_{c}}{P_{c}}=4 \epsilon+6 \epsilon \eta+\frac{3}{2} \eta^{3}+O\left(\eta^{4}, \eta^{2} \epsilon\right),
$$

where $\epsilon=\left(T-T_{c}\right) / T_{c}$ and the order parameter $\eta=-\left(V-V_{c}\right) / V_{c}$.

The above equations of state can be derived from the Gibbs free energy $G$,

$$
G(p, T, \eta)=G_{0}(p, T)+\frac{N}{\rho_{0}^{2}}\left[-(\pi-4 \epsilon) \eta+3 \epsilon \eta^{2}+\frac{3}{8} \eta^{4}\right] .
$$

The similarity of Eq.(46), Eq.(49), and Eq.(51) explains the identical critical exponents in different systems. All systems we considered above can be incorporated into the classical Landau theory [15].

\section{E. Widom Scaling}

Now we consider the scaling symmetry of the free energy around the critical point. We first separate the free energy into two parts,

$$
g(\epsilon, j)=g_{r}(\epsilon, j)+g_{s}(\epsilon, j) .
$$

Function $g_{r}(\epsilon, j)$ is the regular part which does not change when approaching the critical point, while $g_{s}(\epsilon, j)$ is the singular part which possesses the singular behavior of the system in the vicinity of the critical point. Assuming that the singular part is a generalized homogeneous function of its parameters, through Eq. (38), (45), and (46), we can write down the 
free energy near the critical point as

$$
g_{s}(\epsilon, j)=c_{\epsilon} \epsilon^{2}+c_{j} j^{4 / 3}
$$

which has the scaling symmetry,

$$
g_{s}\left(\Lambda^{p} \epsilon, \Lambda^{q} j\right)=\Lambda g_{s}(\epsilon, j), \quad p=\frac{1}{2}, \quad q=\frac{3}{4} .
$$

Following the discussion of [23], we can write the critical exponents in terms of $p$ and $q$ as

$$
\begin{aligned}
& \alpha=\frac{2 p-1}{p}, \\
& \beta=\frac{1-q}{p}, \\
& \gamma=\frac{2 q-1}{p}, \\
& \delta=\frac{q}{1-q} .
\end{aligned}
$$

From the above equations, we find that the critical exponents satisfy the following expected relations,

$$
\begin{gathered}
\alpha+2 \beta+\gamma=2, \\
\alpha+\beta(\delta+1)=2, \\
\gamma(\delta+1)=(2-\alpha)(\delta-1), \\
\gamma=\beta(\delta-1) .
\end{gathered}
$$

The above scaling relations stand as the consistency check for the critical exponents we obtained in the previous section.

\section{F. Phase Diagram}

In FIG 6, we present the phase diagram near critical point $\left(T_{c 1}, J_{c 1}\right)$ on the $T-J$ plane. One can see the phase transition line and the critical point. Liquid/gas-like phase transition occurs when crossing the phase transition line. This phase structure occurs at the temperature above $T_{c 1}$, and ends when the temperature reaches $T_{c 1 \text {, }}$, at which the Maxwell's 


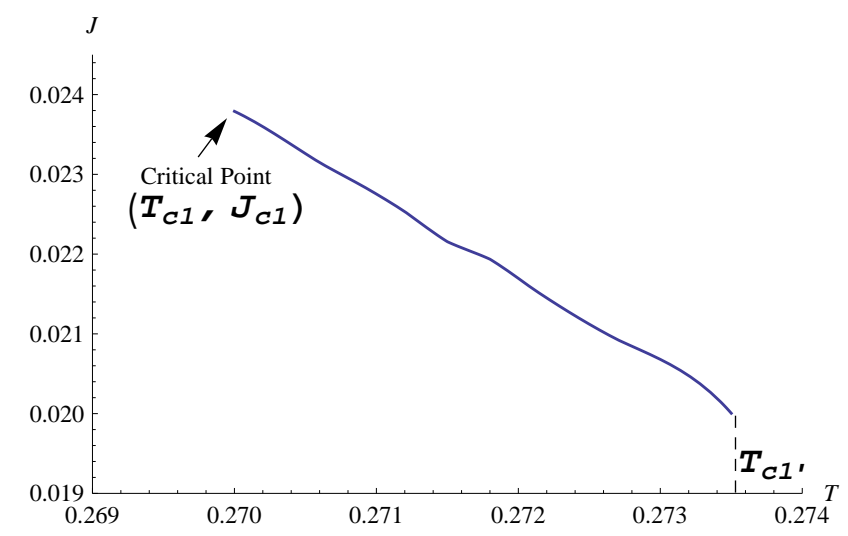

FIG. 6: Phase diagram near critical point $T_{c 1}$. Similar to the phase diagram of liquid/gas phase transition [23, 27].

equal-area law fails to apply on the isotherm due to the stable part is too small.

Although this phase diagram is similar to that of liquid/gas phase transition, this critical phenomenon happens when raising the temperature above $T_{c 1}$, rather than lowering the temperature below the critical temperature. The phase transition line thus point to different direction as that of liquid/gas system [23, 27]. This is an intriguing feature of van der Waalslike critical point for both RN-AdS black hole [25, 30] and Kerr-AdS black hole.

\section{DISCUSSION}

In this paper, we studied the phase structure of Kerr-AdS black hole. Rich critical phenomena have been found at the different temperatures $T_{L}, T_{c 1}$ and $T_{c 2}$. Based on the isotherms, we discussed the physical meaning of each critical point. The asymptotic angular momentum $\Omega_{c 2}$ was also discussed and found to be assoiated to the critical behavior at $T_{c 2}$. We have studied the critical behavior at the critical temperature $T=T_{c 1}$ in a great detail and found the analogy between the critical behavior at $T_{c 1}$ and the van der Waals system. We also calculated the critical exponents. The critical exponents $\left(0, \frac{1}{2}, 1,3\right)$ are the same as that of mean field systems, which means that they are in the same universality class. The corresponding scaling symmetry of free energy has also been discussed. We finally plot the phase diagram of Kerr-AdS black hole.

The multi-critical phenomena we described in this paper could be a guide which provides a restriction for a complete theory of underlying mechanism. We think that the similar van der Waals-like structure, but the difference critical exponents of RN-AdS and Kerr-AdS 
black holes deserves further study. The natural extension, KN-AdS black hole, would be the next target to be understood.

Our results are interesting under the context of AdS/CFT duality. One can study a strong correlation system dual to Kerr-AdS black hole. In [26], Sonner found that superconductinglike condensation also exists on the boundary of KN-AdS black hole. He found that there is a critical value of rotation which could destroy the superconductivity in analogy to the critical magnetic field. It is nature to guess that KN-AdS black hole has the similar phase structure as Kerr-AdS and RN-AdS black holes, which will in some way affect the thermal stability of the condensation. It would be interesting to have further study along this direction.

In recent years, people managed to rewrite the field equations of gravity into thermodynamics identities (for a review see [22]). In [28], Verlinde suggested that the gravity could be understood as the entropy force induced by the inner freedom of the holographic screen. These observations hope to regard gravity theory as a result of more fundamental thermodynamic principles. It could be possible to use the ideas to explain the thermal stability and phase structure of Kerr-AdS black hole.

\section{Acknowledgments}

This work is supported in part by the National Science Council, 50 billions project of Ministry of Education and National Center for Theoretical Science, Taiwan. We thank Professor Tzay-Ming Hong and Professor Ling-Fong Li for fruitful discussions.

[1] Giovanni Arcioni and Ernesto Lozano-Tellechea. Stability and critical phenomena of black holes and black rings. Phys. Rev., D72:104021, 2005.

[2] Rabin Banerjee, Sujoy Kumar Modak, and Saurav Samanta. A New Phase Transition and Thermodynamic Geometry of Kerr- AdS Black Hole. 2010.

[3] Rabin Banerjee, Sujoy Kumar Modak, and Saurav Samanta. New approach to phase transitions in black holes. 2011.

[4] Rong-Gen Cai, Li-Ming Cao, and Ya-Wen Sun. Hawking-Page Phase Transition of black Dp-branes and R-charged black holes with an IR Cutoff. JHEP, 0711:039, 2007. 
[5] Rong-Gen Cai, Zhi-Jiang Lu, and Yuan-Zhong Zhang. Critical behavior in (2+1)-dimensional black holes. Phys.Rev., D55:853-860, 1997.

[6] Rong-Gen Cai and Y.S. Myung. Critical behavior for the dilaton black holes. Nucl.Phys., B495:339-362, 1997.

[7] Rong-Gen Cai and Kwang-Sup Soh. Critical behavior in the rotating D-branes. Mod.Phys.Lett., A14:1895-1908, 1999.

[8] Rong-Gen Cai and Anzhong Wang. Thermodynamics and stability of hyperbolic charged black holes. Phys.Rev., D70:064013, 2004.

[9] Marco M. Caldarelli, Guido Cognola, and Dietmar Klemm. Thermodynamics of KerrNewman-AdS black holes and conformal field theories. Class.Quant.Grav., 17:399-420, 2000.

[10] Steven Carlip and S. Vaidya. Phase transitions and critical behavior for charged black holes. Class.Quant.Grav., 20:3827-3838, 2003.

[11] B. Carter. Hamilton-Jacobi and Schrodinger separable solutions of Einstein's equations. Commun.Math.Phys., 10:280, 1968.

[12] Andrew Chamblin, Roberto Emparan, Clifford V. Johnson, and Robert C. Myers. Holography, thermodynamics and fluctuations of charged AdS black holes. Phys.Rev., D60:104026, 1999.

[13] P.C.W. Davies. THERMODYNAMICS OF BLACK HOLES. Proc.Roy.Soc.Lond., A353:499$521,1977$.

[14] G. W. Gibbons, M. J. Perry, and C. N. Pope. The First Law of Thermodynamics for KerrAnti-de Sitter Black Holes. Class. Quant. Grav., 22:1503-1526, 2005.

[15] N. Goldenfeld. Lectures on phase transitions and the renormalization group. Addison-Wesley Publication Company, 1992.

[16] Sean A. Hartnoll, Christopher P. Herzog, and Gary T. Horowitz. Building a Holographic Superconductor. Phys.Rev.Lett., 101:031601, 2008.

[17] Sean A. Hartnoll, Christopher P. Herzog, and Gary T. Horowitz. Holographic Superconductors. JHEP, 12:015, 2008.

[18] S.W. Hawking and Don N. Page. Thermodynamics of Black Holes in anti-De Sitter Space. Commun.Math.Phys., 87:577, 1983.

[19] C.O. Lousto. The Emergence of an effective two-dimensional quantum description from the study of critical phenomena in black holes. Phys.Rev., D51:1733-1740, 1995.

[20] Juan Martin Maldacena. The large N limit of superconformal field theories and supergravity. 
Adv. Theor. Math. Phys., 2:231-252, 1998.

[21] Makoto Natsuume. Critical phenomena in the AdS/CFT duality. Prog.Theor.Phys.Suppl., 186:491-497, 2010.

[22] T. Padmanabhan. Lessons from Classical Gravity about the Quantum Structure of Spacetime. J. Phys. Conf. Ser., 306:012001, 2011.

[23] R. E. Reichl. A Modern Course in Statistical Physics . University of Texas Press, Austin, TX, 1980.

[24] Anurag Sahay, Tapobrata Sarkar, and Gautam Sengupta. On The Phase Structure and Thermodynamic Geometry of R-Charged Black Holes. JHEP, 1011:125, 2010.

[25] Anurag Sahay, Tapobrata Sarkar, and Gautam Sengupta. Thermodynamic Geometry and Phase Transitions in Kerr- Newman-AdS Black Holes. JHEP, 04:118, 2010.

[26] Julian Sonner. A Rotating Holographic Superconductor. Phys. Rev., D80:084031, 2009.

[27] H. E. Stanley. Introduction to Phase Transitions and Critical Phemonena. Oxford University Press, New York, 1987.

[28] Erik P. Verlinde. On the Origin of Gravity and the Laws of Newton. 2010.

[29] Edward Witten. Anti-de Sitter space and holography. Adv. Theor. Math. Phys., 2:253-291, 1998.

[30] X. N. Wu. Multicritical phenomena of Reissner-Nordstrom anti-de Sitter black holes. Phys.Rev., D62:124023, 2000. 